September 26, 2021

OITS-667

\title{
Determining the Weak Phase $\gamma$ in the Presence of Rescattering [
}

\author{
K. Agashe 2 and N.G. Deshpande 3 \\ Institute of Theoretical Science \\ 5203 University of Oregon \\ Eugene OR 97403-5203
}

\begin{abstract}
We suggest a new technique to determine the CKM phase $\gamma$ without neglecting the (soft) final state rescattering effects. We use (time integrated) $B$ meson decay rates to $\pi$ 's and $K$ 's. A set of $5 \Delta S=0$ (or $1 \Delta S=0$ and $4 \Delta S=1$ ) decay rates is used to compute the strong phases and magnitudes of the tree level and penguin contributions as functions of $\gamma$. These are used to predict a $\Delta S=1(\Delta S=0) B_{d / s}$ decay rate as a function of $\gamma$ (using $S U(3)$ symmetry). The measurement of this decay rate then gives $\gamma$. We illustrate this technique using different cases. Most of the decay modes we use are expected to be accessible at the $B$-factories ( $e^{+} e^{-}$or hadron machines).
\end{abstract}

\footnotetext{
${ }^{1}$ This work is supported by DOE Grant DE-FG03-96ER40969.

${ }^{2}$ email: agashe@oregon.uoregon.edu

${ }^{3}$ email: desh@oregon.uoregon.edu
} 


\section{Introduction}

Determining the angles of the CKM unitarity triangle, denoted by $\alpha, \beta$ and $\gamma$, is one of the important aims of the $B$-factories. Methods have been suggested to determine $\gamma$ (三Arg $\left(-V_{u b}^{\star} V_{u d} / V_{c b}^{\star} V_{c d}\right)$ where $V$ is the CKM matrix) using decays of $B_{d}, B^{+}$and $B_{s}$ (and their CP-conjugates) to two pseudoscalars belonging to the $S U(3)$ octet including the effects of the Electroweak Penguin (EWP) diagrams. These methods rely on the flavor $S U(3)$ symmetry.

Many of these methods neglect the effects of (soft) final state rescattering. In particular, the decay amplitude for $B^{+} \rightarrow \pi^{+} K^{0}$ is assumed to contain only the weak phase $e^{i \pi}$ from the penguin diagram with the top quark in the loop. Tree level operators have the weak phase $e^{i \gamma}$, but since they have the transition $\bar{b} \rightarrow \bar{s} u \bar{u}$, they contribute, in the absence of rescattering, only through annihilation to this decay. Annihilation contributions are argued to be small since they are suppressed by $f_{B} / m_{B}$ (in the absence of significant rescattering effects). Assuming that the $B^{+} \rightarrow \pi^{+} K^{0}$ amplitude has no $e^{i \gamma}$ weak phase, references [1, 2], 3, 4] have suggested methods to determine $\gamma$.

However, rescattering from an intermediate state, for example $\pi^{0} K^{+}$, created by a (color-allowed) spectator decay of a $B^{+}$due to tree level operators, can generate a significant amplitude with the weak phase $e^{i \gamma}$ in the decay $B^{+} \rightarrow \pi^{+} K^{0}$ as well. So it would be better to have a method to determine $\gamma$ which does not use the assumption of no rescattering effects. Rescattering might also enhance annihilation contributions [5] and thus necessitates their inclusion.

Buras and Fleischer [6] gave a method to determine $\gamma$ without neglecting rescattering using $B_{d} \rightarrow \pi^{-} K^{+}, B^{+} \rightarrow \pi^{+} \pi^{0}$ decays and time dependent measurements of the $B_{d} \rightarrow \pi^{0} K_{S}$ decay. For this method, they also require time dependent analysis of, for example, $B_{d} \rightarrow J / \psi K_{S}$ to measure $\beta$. Gronau and Pirjol [7] suggested a method using time independent measurements of all the $B_{d} \rightarrow \pi K$ and $B_{s} \rightarrow \pi K$ modes. In their method also rescattering effects are included. However, it might be diffcult to measure the neutral 


\begin{tabular}{|c|c|c|}
\hline \multirow[b]{2}{*}{ Case } & \multicolumn{2}{|l|}{ Modes used } \\
\hline & $\Delta S=0$ & $\Delta S=1$ \\
\hline 1 & $\begin{aligned} B^{+} \rightarrow & \pi^{+} \pi^{0}, B_{d} \rightarrow \pi^{+} \pi^{-}, \pi^{0} \pi^{0} \\
& \bar{B}_{d} \rightarrow \pi^{+} \pi^{-}, \pi^{0} \pi^{0}\end{aligned}$ & $\begin{array}{c}B_{d} \rightarrow \pi^{-} K^{+}, \pi^{0} K^{0} \\
B_{s} \rightarrow \pi^{+} \pi^{-}\left(\text {or } \pi^{0} \pi^{0}\right)\end{array}$ \\
\hline 2 & $\begin{aligned} B^{+} \rightarrow & \pi^{+} \pi^{0}, B_{d} \rightarrow \pi^{+} \pi^{-}, \pi^{0} \pi^{0} \\
& \bar{B}_{d} \rightarrow \pi^{+} \pi^{-}, \pi^{0} \pi^{0}\end{aligned}$ & $\begin{array}{l}B_{s} \rightarrow K^{+} K^{-} \\
\text {(CP-averaged) }\end{array}$ \\
\hline 3 & $\begin{array}{c}B^{+} \rightarrow \pi^{+} \pi^{0}, B_{d} \rightarrow \pi^{+} \pi^{-}, \pi^{0} \pi^{0} \\
B_{d} \rightarrow K^{+} K^{-}\end{array}$ & $\begin{array}{l}B_{d} \rightarrow \pi^{0} K^{0}, \pi^{-} K^{+} \\
\bar{B}_{d} \rightarrow \pi^{0} \bar{K}^{0}, \pi^{+} K^{-}\end{array}$ \\
\hline 4 & $\begin{array}{c}B^{+} \rightarrow \pi^{+} \pi^{0} \\
B_{s} \rightarrow \pi^{+} K^{-}\left(\text {or } \pi^{0} \bar{K}^{0}\right)(\text { CP-averaged })\end{array}$ & $\begin{array}{l}B_{d} \rightarrow \pi^{0} K^{0}, \pi^{-} K^{+} \\
\bar{B}_{d} \rightarrow \pi^{0} \bar{K}^{0}, \pi^{+} K^{-}\end{array}$ \\
\hline
\end{tabular}

Table 1: The 6 (or 8) $B$ decay modes used by each of the 4 cases to determine $\gamma$

modes of $B_{s}$ decays since that will involve tagging at hadron machines.

In this paper, we suggest a technique to determine $\gamma$ including rescattering effects (and the EWP operators) using $B$ meson decays to $\pi$ 's and $K$ 's. We will illustrate this technique using four cases; see table 1 .

We do not require any time dependent studies. The strategy is as follows. In cases 1 and 2 , using $5 \Delta S=0$ decay modes, we determine the strong phases and magnitudes of the tree level and penguin contributions as functions of $\gamma$ (assuming flavor $S U(2)$ symmetry). Then, using flavor $S U(3)$ symmetry, we predict the rate for one $\Delta S=1$ mode in case 2. In case [4, two $\Delta S=1$ modes have to be measured to make a prediction for a third $\Delta S=1$ mode. The measurement of the decay for which we have a prediction (as a function of $\gamma$ ) then determines $\gamma$. A similar idea can be applied to predict a $\Delta S=0$ decay mode as a function of $\gamma$ using measurements of $\Delta S=1$ (and some $\Delta S=0$ ) modes (cases 3 and 田).

The $B^{+} \rightarrow \pi^{+} \pi^{0}, B_{d} \rightarrow \pi \pi, \pi K$ modes should be relatively accessible at the $e^{+} e^{-}$and hadron machines. For the $B_{d}$ decays to a CP eigenstate, we require (external) tagging (i.e., the $\mathrm{CP}$-averaged decay rate is not sufficient). The $B_{s}$ decay modes were accessible at LEP1 and will be accessible at hadron machines. The $B_{s} \rightarrow \pi \pi$ decay mode (case 1) might be hard to measure since 
it requies tagging whereas in cases 2 and 4 , the $B_{s}$ modes are either "selftagging" $\left(\pi^{+} K^{-}\right)$or a CP-averaged measurement is sufficient (for $K^{-} K^{+}$, $\left.\pi^{0} \bar{K}^{0}\right)$. In case 1 , we show that if we measure additional $B_{d}$ modes, a CPaveraged measurement of the decay rate $B_{s} \rightarrow \pi \pi$ is sufficient.

Although flavor $S U(3)$ symmetry is used in all the four cases (as in all the other methods mentioned above), in the last section, we discuss how to take into account $S U(3)$ breaking.

\section{Cases 1 and 2}

We will write the decay amplitudes for the decays $B_{i} \rightarrow M M$, where $M$ is a pseudoscalar belonging to the flavor $S U(3)$ octet, in terms of the 5 linearly independent $S U(3)$ invariant amplitudes denoted by $C_{3}^{T, P}, C_{6}^{T, P}, C_{15}^{T, P}, A_{3}^{T, P}$ and $A_{15}^{T, P}$ (where $T$ and $P$ stand for the parts of these amplitudes generated by tree level and penguin operators, respectively). These $S U(3)$ invariant amplitudes include rescattering effects. The annihilation amplitudes, $A_{3,15}$, are the ones in which the quark index $i$ of $B_{i}$ is contracted directly with the Hamiltonian. Neglecting rescattering effects is equivalent to assuming $C_{3}^{T}-C_{6}^{T}-C_{15}^{T}=0$ and $A_{3,15} \sim f_{B} / m_{B}$. For example, the tree level part of the decay amplitude $\mathcal{A}\left(B^{+} \rightarrow \pi^{+} K^{0}\right)$ contains this combination of the $C^{T}$ amplitudes and $A_{15}^{T}$.

In this notation [8], the amplitudes for $B \rightarrow \pi \pi$ decays can be written as

$$
\begin{aligned}
-\sqrt{2} \mathcal{A}\left(B^{+} \rightarrow \pi^{+} \pi^{0}\right)= & 8\left(\lambda_{u}^{(d)} C_{15}^{T}+\sum_{q} \lambda_{q}^{(d)} C_{15, q}^{P}\right) \\
= & -3 I_{2} \\
\sqrt{2} \mathcal{A}\left(B_{d} \rightarrow \pi^{0} \pi^{0}\right)= & \lambda_{u}^{(d)}\left(C_{3}^{T}+C_{6}^{T}-5 C_{15}^{T}\right)+\sum_{q} \lambda_{q}^{(d)}\left(C_{3, q}^{P}+C_{6, q}^{P}-5 C_{15, q}^{P}\right) \\
& +\lambda_{u}^{(d)}\left(2 A_{3}^{T}+A_{15}^{T}\right)+\sum_{q} \lambda_{q}^{(d)}\left(2 A_{3, q}^{P}+A_{15, q}^{P}\right) \\
= & -I_{0}+2 I_{2},
\end{aligned}
$$




$$
\begin{aligned}
\mathcal{A}\left(B_{d} \rightarrow \pi^{+} \pi^{-}\right)= & -\lambda_{u}^{(d)}\left(C_{3}^{T}+C_{6}^{T}+3 C_{15}^{T}\right)-\sum_{q} \lambda_{q}^{(d)}\left(C_{3, q}^{P}+C_{6, q}^{P}+3 C_{15, q}^{P}\right) \\
& -\lambda_{u}^{(d)}\left(2 A_{3}^{T}+A_{15}^{T}\right)-\sum_{q} \lambda_{q}^{(d)}\left(2 A_{3, q}^{P}+A_{15, q}^{P}\right) \\
= & I_{0}+I_{2} .
\end{aligned}
$$

Here, $\lambda_{q}^{\left(q^{\prime}\right)}=V_{q b}^{\star} V_{q q^{\prime}}\left(q=u, c, t\right.$ and $\left.q^{\prime}=d, s\right)$ and $C_{q}^{P}, A_{q}^{P}$ denote the penguin amplitudes due to $q$ running in the loop. f $I_{2}$ and $I_{0}$ are the amplitudes for $B \rightarrow \pi \pi(I=2)$ and $(I=0)$ respectively or in other words the $\Delta I=3 / 2$ and $\Delta I=1 / 2$ amplitudes.

Using the unitarity of the CKM matrix, i.e., $\lambda_{t}^{(d)}=-\lambda_{u}^{(d)}-\lambda_{c}^{(d)}$, we get

$$
\lambda_{u}^{(d)} C_{i}^{T}+\sum_{q} \lambda_{q}^{(d)} C_{i, q}^{P}=\lambda_{u}^{(d)} \tilde{C}_{i}^{T}-\lambda_{c}^{(d)} C_{i}^{P}
$$

where $\tilde{C}_{i}^{T}=C_{i}^{T}-C_{i, t}^{P}+C_{i, u}^{P}$ and $C_{i}^{P}=C_{i, t}^{P}-C_{i, c}^{P}$. A similar notation is used for $\tilde{A}_{i}^{T}$ and $A_{i}^{P}$.

In the $B^{+} \rightarrow \pi^{+} \pi^{0}$ decay, which contains only the $\Delta I=3 / 2$ amplitude, there is no contribution to $C_{15, q}^{P}$ from the strong penguin diagrams since these diagrams are $\Delta I=1 / 2$. Neubert and Rosner [9] showed that $C_{15, q}^{P}=$ $C_{15}^{T} 3 / 2 \kappa_{q}$, where $\kappa_{q}=\left(c_{9, q}+c_{10, q}\right) /\left(c_{1}+c_{2}\right)$ is the ratio of Wilson coefficients (WC's) of the EWP operators (with quark $q$ running in the loop) and the tree level operators in the effective Hamiltonian. We expect $c_{(9,10), t} \gg c_{(9,10),(u, c)}$ since the top quark EWP diagram with $Z$ exchange is enhanced by $m_{t}^{2} / m_{Z}^{2}$ and so henceforth we neglect $\kappa_{u, c}$ and denote $\kappa_{t}$ by $\kappa$. So, we get

$$
\begin{gathered}
\tilde{C}_{15}^{T} \approx C_{15}^{T}\left(1-\frac{3}{2} \kappa\right), \\
C_{15}^{P} \approx C_{15}^{T} \frac{3}{2} \kappa
\end{gathered}
$$

\footnotetext{
${ }^{4}$ Tree level operators with the flavor structure $c \bar{c} \bar{b} d$ can also contribute through rescattering from charm intermediate states. This rescattering generates a charm-quark penguin topology with the amplitude being proportional to $\lambda_{c}^{(d)}$ and so can be included as part of $C_{i, c}^{P}$.
} 
Using Eqns.(44), (5) and (6), we can rewrite $-3 I_{2}=8\left(\lambda_{u}^{(d)} C_{15}^{T}+\sum_{q} \lambda_{q}^{(d)} C_{15, q}^{P}\right)$ $=8\left(\lambda_{u}^{(d)} \tilde{C}_{15}^{T}-\lambda_{c}^{(d)} C_{15}^{P}\right)$ as

$$
-3 I_{2} \approx 8 \tilde{C}_{15}^{T}\left(\lambda_{u}^{(d)}-\frac{\frac{3}{2} \kappa}{1-\frac{3}{2} \kappa} \lambda_{c}^{(d)}\right) .
$$

Since $3 / 2 \kappa \sim 2 \%$ and $\left|\lambda_{u}^{(d)}\right| \sim\left|\lambda_{c}^{(d)}\right|$, we neglect the second term (i.e., the EWP contribution) in the right hand side of Eqn.(7) for now and we assume $\left|\tilde{C}_{15}^{T}\right| \approx\left|C_{15}^{T}\right|$. We will show later how to include it. Then, using the Wolfenstein parametrization in which $\lambda_{u}^{(d)}=\left|\lambda_{u}^{(d)}\right| e^{i \gamma}$ and $\lambda_{c}^{(d)}=\left|\lambda_{c}^{(d)}\right|$ (and similarly for $d$ replaced by $s$ ), we get

$$
-3 I_{2} \approx\left|\lambda_{u}^{(d)}\right| e^{i \gamma} 8\left|\tilde{C}_{15}^{T}\right| \approx\left|\lambda_{u}^{(d)}\right| e^{i \gamma} 8\left|C_{15}^{T}\right|
$$

so that $\left|C_{15}^{T}\right|$ can be obtained directly from the $B^{+} \rightarrow \pi^{+} \pi^{0}$ decay rate. We have chosen a phase convention such that the strong phase of $C_{15}^{T}$ is zero.

From Eqns.(2) and (3), we get

$$
\begin{aligned}
I_{0}= & -\lambda_{u}^{(d)}\left(\tilde{C}_{3}^{T}+\tilde{C}_{6}^{T}+\frac{1}{3} \tilde{C}_{15}^{T}\right)+\lambda_{c}^{(d)}\left(C_{3}^{P}+C_{6}^{P}+\frac{1}{3} C_{15}^{P}\right) \\
& -\lambda_{u}^{(d)}\left(2 \tilde{A}_{3}^{T}+\tilde{A}_{15}^{T}\right)+\lambda_{c}^{(d)}\left(2 A_{3}^{P}+A_{15}^{P}\right) \\
\equiv & e^{i \phi_{\tilde{T}}}\left|\lambda_{u}^{(d)}\right| e^{i \gamma} \tilde{T}-\left|\lambda_{c}^{(d)}\right| e^{i \phi_{P}} P .
\end{aligned}
$$

The five quantities: $\left|C_{15}^{T}\right|, \tilde{T}, P, \phi_{\tilde{T}}$ and $\phi_{P}$ (where the $\phi$ 's are the CP conserving strong phases) can thus be determined as functions of $\gamma$ from the measurements of the five rates: $B^{+} \rightarrow \pi^{+} \pi^{0}, B_{d} \rightarrow \pi^{+} \pi^{-}, B_{d} \rightarrow \pi^{0} \pi^{0}$ and the CP-conjugates of the $B_{d}$ decays.

Explicitly, rotating the CP-conjugate amplitudes by $e^{i 2 \gamma}$ (and denoting them by "bars"), we get the triangle formed by $B^{+} \rightarrow \pi^{+} \pi^{0}, B_{d} \rightarrow \pi^{+} \pi^{-}$ and $B_{d} \rightarrow \pi^{0} \pi^{0}$ (Eqns.(11), (2) and (3)):

$$
-\sqrt{2} \mathcal{A}\left(B^{+} \rightarrow \pi^{+} \pi^{0}\right)+\sqrt{2} \mathcal{A}\left(B_{d} \rightarrow \pi^{0} \pi^{0}\right)+\mathcal{A}\left(B_{d} \rightarrow \pi^{+} \pi^{-}\right)=0
$$

and the one formed by the CP-conjugate decays. These are shown in Fig.1 (from Eqn.(8), $I_{2}=\bar{I}_{2}$ ). 


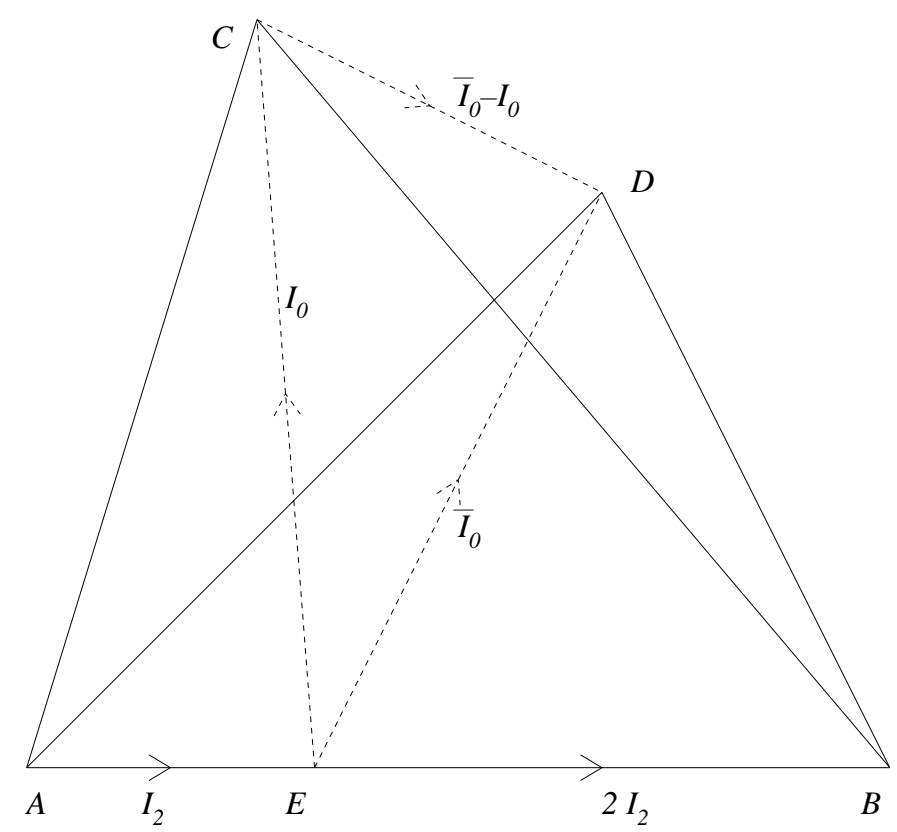

Figure 1: The triangles formed by the $B \rightarrow \pi \pi$ amplitudes: $A B=$ $\left|\sqrt{2} \mathcal{A}\left(B^{+} \rightarrow \pi^{+} \pi^{0}\right)\right|, A C=\left|\mathcal{A}\left(B_{d} \rightarrow \pi^{+} \pi^{-}\right)\right|, B C=\left|\sqrt{2} \mathcal{A}\left(B_{d} \rightarrow \pi^{0} \pi^{0}\right)\right|$, $A D=\left|\mathcal{A}\left(\bar{B}_{d} \rightarrow \pi^{+} \pi^{-}\right)\right|$and $B D=\left|\sqrt{2} \mathcal{A}\left(\bar{B}_{d} \rightarrow \pi^{0} \pi^{0}\right)\right|$. In the phase convention where the strong phase of $C_{15}^{T}$ is zero, the angle between $I_{2}$ and the real axis (not shown) is $\pi+\gamma$ (see Eqn.(8)).

From Eqn.(99), we get

$$
\bar{I}_{0}-I_{0}=-\left|\lambda_{c}^{(d)}\right| e^{i \phi_{P}} P\left(e^{i 2 \gamma}-1\right) .
$$

Thus, the length of $\bar{I}_{0}-I_{0}$ (obtained from Fig.1) gives $P$ as a function of $\gamma$. The angle between $\bar{I}_{0}-I_{0}$ and $I_{2}$ is $\phi_{P}+\pi / 2$ (see Eqns.(8) and (11)) so that the orientation of $\bar{I}_{0}-I_{0}$ (obtained from Fig.(1) gives $\phi_{P}$ independent of $\gamma$. 回

Similarly,

$$
\bar{I}_{0} e^{-i 2 \gamma}-I_{0}=\left|\lambda_{u}^{(d)}\right| e^{i \phi_{\tilde{T}} \tilde{T}}\left(e^{-i \gamma}-e^{i \gamma}\right)
$$

gives $\phi_{\tilde{T}}$ and $\tilde{T}$ (or knowing $I_{0}, P$ and $\phi_{P}$ gives $\phi_{\tilde{T}}$ and $\tilde{T}$ using Eqn.(9)).

There is a discrete ambiguity in this procedure since in Fig.11, the vertices $C$ and $D$ could be on opposite sides of $I_{2}$. This has been discussed in the literature [10] [11].

\footnotetext{
5 This was also discussed in 10$]$.
} 
All the analysis up to now actually relies only on flavor $S U(2)$ symmetry.

\subsection{Case 1}

The $B_{d} \rightarrow K \pi$ and $B_{s} \rightarrow \pi \pi$ amplitudes are given by [ $]$

$$
\begin{gathered}
-\mathcal{A}\left(B_{d} \rightarrow K^{+} \pi^{-}\right)=\lambda_{u}^{(s)}\left(C_{3}^{T}+C_{6}^{T}+3 C_{15}^{T}\right)+\sum_{q} \lambda_{q}^{(s)}\left(C_{3, q}^{P}+C_{6, q}^{P}+3 C_{15, q}^{P}\right) \\
-\lambda_{u}^{(s)} A_{15}^{T}-\sum_{q} \lambda_{q}^{(s)} A_{15, q}^{P} \\
\sqrt{2} \mathcal{A}\left(B_{d} \rightarrow \pi^{0} K^{0}\right)= \\
\lambda_{u}^{(s)}\left(C_{3}^{T}+C_{6}^{T}-5 C_{15}^{T}\right)+\sum_{q} \lambda_{q}^{(s)}\left(C_{3, q}^{P}+C_{6, q}^{P}-5 C_{15, q}^{P}\right) \\
-\lambda_{u}^{(s)} A_{15}^{T}-\sum_{q} \lambda_{q}^{(s)} A_{15, q}^{P} \\
\begin{aligned}
2 \mathcal{A}\left(B_{s} \rightarrow \pi^{+} \pi^{-}\right)= & \mathcal{A}\left(B_{s} \rightarrow \pi^{0} \pi^{0}\right) \\
= & -\lambda_{u}^{(s)}\left(4 A_{3}^{T}+4 A_{15}^{T}\right) \\
& -\sum_{q} \lambda_{q}^{(s)}\left(4 A_{3, q}^{P}+4 A_{15, q}^{P}\right) .
\end{aligned}
\end{gathered}
$$

From Eqns.(13) and (15) and using the notation of Eqn.(14) and rearranging we get

$$
\begin{aligned}
\mathcal{A}\left(B_{d} \rightarrow K^{+} \pi^{-}\right)+\mathcal{A}\left(B_{s} \rightarrow \pi^{+} \pi^{-}\right)= & -\lambda_{u}^{(s)}\left(\tilde{C}_{3}^{T}+\tilde{C}_{6}^{T}+\frac{1}{3} \tilde{C}_{15}^{T}\right) \\
& +\lambda_{c}^{(s)}\left(C_{3}^{P}+C_{6}^{P}+\frac{1}{3} C_{15}^{P}\right) \\
& -\lambda_{u}^{(s)}\left(2 \tilde{A}_{3}^{T}+\tilde{A}_{15}^{T}\right)+\lambda_{c}^{(s)}\left(2 A_{3}^{P}+A_{15}^{P}\right) \\
& -\frac{8}{3}\left(\lambda_{u}^{(s)} \tilde{C}_{15}^{T}-\lambda_{c}^{(s)} C_{15}^{P}\right) .
\end{aligned}
$$

Using Eqns.(6) and (9) and assuming $C_{15}^{T} \approx \tilde{C}_{15}^{T}$, we get

$$
\begin{aligned}
\mathcal{A}\left(B_{d} \rightarrow K^{+} \pi^{-}\right)+\mathcal{A}\left(B_{s} \rightarrow \pi^{+} \pi^{-}\right)= & e^{i \phi_{\tilde{T}}}\left|\lambda_{u}^{(s)}\right| e^{i \gamma} \tilde{T}-\left|\lambda_{c}^{(s)}\right| e^{i \phi_{P}} P \\
& -\frac{8}{3} C_{15}^{T}\left(\lambda_{u}^{(s)}-\frac{3}{2} \kappa \lambda_{c}^{(s)}\right),
\end{aligned}
$$


i.e., from Eqns.(3) and (16), we see that the combination of the amplitudes $\mathcal{A}\left(B_{d} \rightarrow K^{+} \pi^{-}\right)+\mathcal{A}\left(B_{s} \rightarrow \pi^{+} \pi^{-}\right)$can be obtained from the amplitude for $B_{d} \rightarrow \pi^{+} \pi^{-}$by scaling the tree level contribution in the latter by $\left|\lambda_{u}^{(s)}\right| /\left|\lambda_{u}^{(d)}\right|$ and the penguin contribution by $\left|\lambda_{c}^{(s)}\right| /\left|\lambda_{c}^{(d)}\right|$. In particular the EWP contribution $\left(\propto \lambda_{c}^{(s)}\right)$ is important in the last line of Eqn.(17) since, due to the CKM factors, it is comparable to the tree level contribution (unlike in the $B_{d} \rightarrow \pi^{+} \pi^{-}$decay; see Eqn.(7) .

Similarly, from Eqns.(2), (14) and (15), we see that the combination of the amplitudes $\sqrt{2} \mathcal{A}\left(B_{d} \rightarrow K^{0} \pi^{0}\right)-\mathcal{A}\left(B_{s} \rightarrow \pi^{-} \pi^{+}\right)$can be obtained from the amplitude $\sqrt{2} \mathcal{A}\left(B_{d} \rightarrow \pi^{0} \pi^{0}\right)$ by scaling the latter by CKM factors. This gives

$$
\begin{aligned}
\sqrt{2} \mathcal{A}\left(B_{d} \rightarrow K^{0} \pi^{0}\right)-\mathcal{A}\left(B_{s} \rightarrow \pi^{+} \pi^{-}\right)= & -e^{i \phi_{\tilde{T}}}\left|\lambda_{u}^{(s)}\right| e^{i \gamma} \tilde{T}+\left|\lambda_{c}^{(s)}\right| e^{i \phi_{P}} P \\
& -\frac{16}{3} C_{15}^{T}\left(\lambda_{u}^{(s)}-\frac{3}{2} \kappa \lambda_{c}^{(s)}\right) .(18)
\end{aligned}
$$

We emphasize again that in obtaining the last lines of Eqns.(17) and (18), it is crucial that we use the Neubert-Rosner result (Eqn.(6)), i.e., that $\kappa$ is calculable. If $\mathcal{A}\left(B_{s} \rightarrow \pi^{+} \pi^{-}\right) \equiv a^{\prime} e^{i \phi_{a}^{\prime}}$, we can determine $a^{\prime}$ from the decay rate $B_{s} \rightarrow \pi^{+} \pi^{-}$. Then, measuring the decay rate $B_{d} \rightarrow \pi^{-} K^{+}$gives $\phi_{a}^{\prime}$ as a function of $\gamma$ (using Eqn.(17)) $\left(\phi_{P}, \phi_{\tilde{T}}, P\right.$ and $\tilde{T}$ are already known as functions of $\gamma$ ). Knowing $a^{\prime}$ and $\phi_{a}^{\prime}$, we have a prediction for the decay rate $B_{d} \rightarrow K^{0} \pi^{0}$ (Eqn.(18)) and then $\gamma$ can be determined by measuring this decay. I Thus, we can determine $\gamma$, including rescattering effects, by measuring the 8 decay modes: $B^{+} \rightarrow \pi^{+} \pi^{0}, B_{d}$ and $\bar{B}_{d} \rightarrow \pi^{+} \pi^{-}, \pi^{0} \pi^{0}$, $B_{d} \rightarrow \pi^{-} K^{+}, B_{d} \rightarrow K^{0} \pi^{0}$ and $B_{s} \rightarrow \pi \pi$ (any one) (or CP-conjugates of the last three modes).

From Eqn.(15), we see that the decay mode $B_{s} \rightarrow \pi \pi$ has only an annihilation contribution. If (either from experimental measurement of the $B_{s} \rightarrow \pi \pi$ rate (or a limit on the rate) or a theoretical estimate including

\footnotetext{
${ }^{6}$ In this part of the technique, we introduce two additional discrete ambiguities - one in determining $\phi_{a}^{\prime}$ and another in the final determination of $\gamma$ from the $B_{d} \rightarrow K^{0} \pi^{0}$ rate.
} 
rescattering) the annihilation amplitude $\sim A_{3}+A_{15}$ does turn out to be small (smaller than, say, the experimental error in the measurement of the (magnitude) of $\left.\mathcal{A}\left(B_{d} \rightarrow \pi K\right)\right)$ then, a decay rate $B_{d} \rightarrow \pi K$ can be predicted (as a function of $\gamma$ ) by simply scaling the corresponding $B_{d} \rightarrow \pi \pi$ amplitude by CKM factors. Thus, in this case, 6 decay modes: $B^{+} \rightarrow \pi^{+} \pi^{0}, B_{d}$ (and $\left.\bar{B}_{d}\right) \rightarrow \pi^{+} \pi^{-}, \pi^{0} \pi^{0}$ and any one $B_{d} \rightarrow \pi K$ are sufficient to determine $\gamma$.

As mentioned in the introduction, we require tagging to measure the $B_{s} \rightarrow \pi \pi$ decay mode (i.e., the $\mathrm{CP}$-averaged rate is not sufficient in the above method). If this tagged decay rate is hard to measure whereas the $\mathrm{CP}$-averaged rate can be measured, we can proceed as follows. Writing $\mathcal{A}\left(\bar{B}_{s} \rightarrow \pi^{+} \pi^{-}\right) \equiv \bar{a}^{\prime} e^{\bar{\phi}_{a}^{\prime}}$, the CP-averaged rate is $1 / 2\left(a^{\prime 2}+\bar{a}^{\prime 2}\right)$. Measuring the $B_{d} \rightarrow \pi K$ decay rates, from Eqns.(17) and (18), we can determine $a^{\prime}$ (and $\phi_{a}^{\prime}$ ) as a function of $\gamma$. Similarly, the $\bar{B}_{d} \rightarrow \pi K$ decay rates will give $\bar{a}^{\prime}$. Thus, the CP-averaged $B_{s} \rightarrow \pi \pi$ rate can be predicted as a function of $\gamma$ and its measurement gives $\gamma$.

\subsection{Case 2}

The expression for the $B_{s} \rightarrow K^{+} K^{-}$decay amplitude in terms of the $S U(3)$ invariant amplitudes is identical to that for $B_{d} \rightarrow \pi^{-} \pi^{+}$, including annihilation contributions (unlike the decay mode $B_{d} \rightarrow \pi^{-} K^{+}$), modulo CKM factors, i.e., up to $\lambda_{q}^{(d)} \rightarrow \lambda_{q}^{(s)}$ [8]. Thus, we also have a prediction (as a function of $\gamma$ ) for this decay rate and also the rate for its CP-conjugate process including all rescattering effects. So, the measurement of this $\mathrm{CP}$-averaged decay rate can be used to determine $\gamma$.

\section{Cases 3 and 4}

Using the same technique as in section 2, we can predict the rate for a $B_{d} \rightarrow$ $\pi \pi\left(\right.$ or a $B_{s} \rightarrow \pi K$ ) decay as a function of $\gamma$ given the decay rates for $B_{d} \rightarrow \pi K$. The difference is that in the triangle construction to determine 
the tree level and penguin contributions to the $B_{d} \rightarrow \pi K$ amplitudes, we have to take into account the EWP contributions to the $B_{d} \rightarrow \pi K$ amplitudes (the EWP amplitudes were neglected in the $B \rightarrow \pi \pi$ triangles in section 2; see Eqns.(17) and (8)). I So, we discuss the application of the technique again.

The decay amplitudes for $B_{d} \rightarrow \pi K$ (Eqns.(13) and (14)) can be written as

$$
\begin{aligned}
\sqrt{2} \mathcal{A}\left(B_{d} \rightarrow \pi^{0} K^{0}\right) & =-I_{1 / 2}+2 I_{3 / 2} \\
\mathcal{A}\left(B_{d} \rightarrow \pi^{-} K^{+}\right) & =I_{1 / 2}+I_{3 / 2}
\end{aligned}
$$

where $I_{1 / 2}$ and $I_{3 / 2}$ are the amplitudes for $B_{d}$ decay to $\pi K(I=1 / 2)$ and $(I=3 / 2)$ respectively. Then,

$$
\begin{aligned}
3 I_{3 / 2} & =\sqrt{2} \mathcal{A}\left(B_{d} \rightarrow \pi^{0} K^{0}\right)+\mathcal{A}\left(B_{d} \rightarrow \pi^{-} K^{+}\right) \\
& =-\lambda_{u}^{(s)} 8 \tilde{C}_{15}^{T}+8 \lambda_{c}^{(s)} C_{15}^{P} \\
& =-8 C_{15}^{T}\left(\lambda_{u}^{(s)}-\frac{3}{2} \kappa \lambda_{c}^{(s)}\right) \\
& =-8\left|C_{15}^{T}\right|\left|\lambda_{u}^{(s)}\right|\left(e^{i \gamma}+\delta_{E W}\right)
\end{aligned}
$$

using Eqn.(6) and $\left|\tilde{C}_{15}^{T}\right| \approx\left|C_{15}^{T}\right|$ (i.e., neglecting the EWP contribution in the $B^{+} \rightarrow \pi^{+} \pi^{0}$ decay). $\delta_{E W}$ is given by $-\left|\lambda_{c}^{(s)}\right| /\left|\lambda_{u}^{(s)}\right| 3 / 2 \kappa \sim O(1)$, i.e., as mentioned earlier, the EWP contribution is important for $B_{d} \rightarrow \pi K$ decays. $\left|C_{15}^{T}\right|$ can be obtained from the $B^{+} \rightarrow \pi^{+} \pi^{0}$ decay rate as before.

$I_{1 / 2}$ is given by (in analogy to $I_{0}$ of section 2)

$$
\begin{aligned}
I_{1 / 2}= & -\lambda_{u}^{(s)}\left(\tilde{C}_{3}^{T}+\tilde{C}_{6}^{T}+\frac{1}{3} \tilde{C}_{15}^{T}\right)+\lambda_{c}^{(s)}\left(C_{3}^{P}+C_{6}^{P}+\frac{1}{3} C_{15}^{P}\right) \\
& +\lambda_{u}^{(s)} \tilde{A}_{15}^{T}-\lambda_{c}^{(s)} A_{15}^{P} \\
\equiv & e^{i \phi_{\tilde{T}}^{\prime}}\left|\lambda_{u}^{(s)}\right| e^{i \gamma} \tilde{T}^{\prime}-\left|\lambda_{c}^{(s)}\right| e^{i \phi_{P}^{\prime}} P^{\prime} .
\end{aligned}
$$

As in section Q 2 , the four quantities: $\tilde{T}^{\prime}, P^{\prime}, \phi_{\tilde{T}}^{\prime}$ and $\phi_{P}^{\prime}$ can thus be determined as functions of $\gamma$ from the measurements of the four decay rates:

\footnotetext{
${ }^{7}$ Of course, in section 2, to make a prediction for $\Delta S=1$ decay modes, we did have to include the EWP contribution to the $\Delta S=1$ decay amplitudes.
} 
$B_{d} \rightarrow \pi^{-} K^{+}, B_{d} \rightarrow \pi^{0} K^{0}$ and their CP-conjugates. P

Due to the EWP contribution (see Eqn.(21)), the triangle construction is a bit different in this case as shown below.

As before, we multiply the CP-conjugate amplitudes by $e^{i 2 \gamma}$ to get the "barred" amplitudes. In this case (unlike the case for $I_{2}$ in section 2) there is an angle between $I_{3 / 2}$ and $\bar{I}_{3 / 2}$ denoted by $2 \tilde{\gamma}$ and their magnitudes are functions of $\gamma$ (see Eqn.(21)):

$$
\begin{gathered}
\left|I_{3 / 2}\right|=\left|\bar{I}_{3 / 2}\right|=\frac{8}{3}\left|C_{15}^{T}\right|\left|\lambda_{u}^{(s)}\right| \sqrt{\left(1+\delta_{E W}^{2}+2 \delta_{E W} \cos \gamma\right)} \\
\tan \tilde{\gamma}=\frac{\delta_{E W} \sin \gamma}{1+\delta_{E W} \cos \gamma} .
\end{gathered}
$$

Given $\gamma$, we can thus construct the triangles of Eqn.(21) and its CPconjugate (see Fig.21). P As in section 2, knowing the magnitudes and orientations of $I_{1 / 2}$ and $\bar{I}_{1 / 2}$ from Fig.2, we can determine $\tilde{T}^{\prime}, P^{\prime}, \phi_{\tilde{T}}^{\prime}$ and $\phi_{P}^{\prime}$ as functions of $\gamma$, using equations similar to Eqns.(11) and (12).

This construction also shows how to include the EWP contributions to the $B^{+} \rightarrow \pi^{+} \pi^{0}$ decay in section 2 as follows.

Once EWP's are included, as for the case of $I_{3 / 2}$ and $\bar{I}_{3 / 2}$, there is an angle $2 \tilde{\gamma}^{\prime}$ between $I_{2}$ and $\bar{I}_{2}$ and the magnitude of $\tilde{C}_{15}^{T}$ will depend on $\gamma$ (see Eqn.(7)):

$$
\begin{aligned}
3\left|I_{2}\right|=3\left|\bar{I}_{2}\right|= & \left|\sqrt{2} \mathcal{A}\left(B^{+} \rightarrow \pi^{+} \pi^{0}\right)\right| \\
= & 8\left|\tilde{C}_{15}^{T}\right|\left|\lambda_{u}^{(d)}\right| \sqrt{\left(1+\delta_{E W}^{\prime 2}+2 \delta_{E W}^{\prime} \cos \gamma\right)} \\
& \tan \tilde{\gamma}^{\prime}=\frac{\delta_{E W}^{\prime} \sin \gamma}{1+\delta_{E W}^{\prime} \cos \gamma},
\end{aligned}
$$

where $\delta_{E W}^{\prime}$ is given by $-\left|\lambda_{c}^{(d)}\right| /\left|\lambda_{u}^{(d)}\right| 3 / 2 \kappa /(1-3 / 2 \kappa) \sim O($ few $\%)$.

\footnotetext{
${ }^{8} \mathrm{~A}$ simlilar analysis can be done with the $B^{+} \rightarrow \pi K$ decay amplitudes which can be written in terms of $I_{3 / 2}$ (the same as for $B_{d} \rightarrow \pi K$ decays) and $I_{1 / 2}^{\prime}$ which is a different combination of the $S U(3)$ invariant amplitudes than $I_{1 / 2}$. Thus, the $B^{+} \rightarrow \pi K$ decay rates are not useful as far as predicting the $B_{d} \rightarrow \pi \pi$ (case 3) (or $B_{s} \rightarrow \pi K$ in case 1) decay rates is concerned.

${ }^{9}$ As in section 2, there is a discrete ambiguity in the orientation of the triangles.
} 


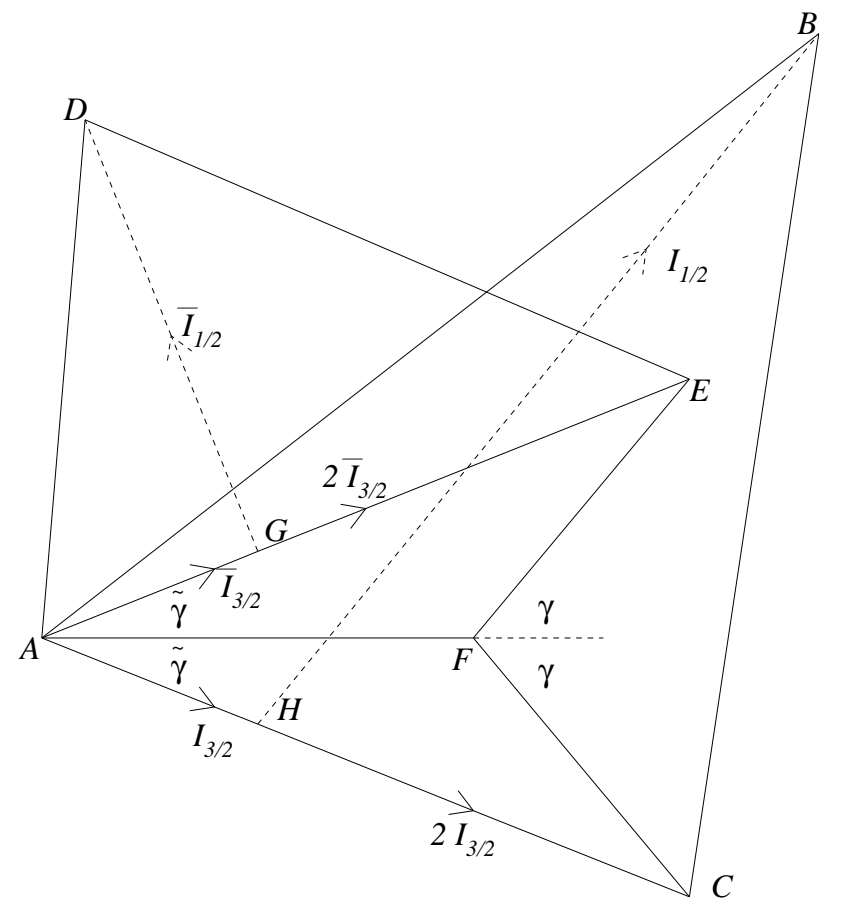

Figure 2: The triangles formed by the $B_{d} \rightarrow \pi K$ amplitudes: $A B=$ $\left|\mathcal{A}\left(B_{d} \rightarrow K^{+} \pi^{-}\right)\right|, B C=\left|\sqrt{2} \mathcal{A}\left(B_{d} \rightarrow \pi^{0} K^{0}\right)\right|, A D=\left|\mathcal{A}\left(\bar{B}_{d} \rightarrow K^{-} \pi^{+}\right)\right|$, $D E=\left|\sqrt{2} \mathcal{A}\left(\bar{B}_{d} \rightarrow \pi^{0} \bar{K}^{0}\right)\right| . \quad A F=\left|\sqrt{2} \mathcal{A}\left(B^{+} \rightarrow \pi^{+} \pi^{0}\right)\right|\left|\lambda_{u}^{(s)}\right| /\left|\lambda_{u}^{(d)}\right|$ and $F C=F E=A F \delta_{E W}$ (see Eqn.(21)). As in Fig.11, in the phase convention where the strong phase of $C_{15}^{T}$ is zero, the angle between $A F$ and the real axis is $\pi+\gamma$.

\subsection{Case 3}

The $B_{d} \rightarrow K^{+} K^{-}$amplitude is given by [Q]

$$
\begin{aligned}
\mathcal{A}\left(B_{d} \rightarrow K^{+} K^{-}\right) & =-\lambda_{u}^{(d)}\left(2 A_{3}^{T}+2 A_{15}^{T}\right)-\sum_{q} \lambda_{q}^{(d)}\left(2 A_{3, q}^{P}+2 A_{15, q}^{P}\right) \\
& \equiv a e^{i \phi_{a}} .
\end{aligned}
$$

From Eqns.(2), (3), (13), (14) and (27), we can see that $\sqrt{2} \mathcal{A}\left(B_{d} \rightarrow \pi^{0} \pi^{0}\right)+$ $\mathcal{A}\left(B_{d} \rightarrow K^{-} K^{+}\right)$can be obtained from $\sqrt{2} \mathcal{A}\left(B_{d} \rightarrow K^{0} \pi^{0}\right)$ and $\mathcal{A}\left(B_{d} \rightarrow \pi^{+} \pi^{-}\right)-\mathcal{A}\left(B_{d} \rightarrow K^{-} K^{+}\right)$can be obtained from $\mathcal{A}\left(B_{d} \rightarrow \pi^{-} K^{+}\right)$ by scaling the $\Delta S=1$ amplitudes by appropriate CKM factors, i.e., in this case, the decay mode $B_{d} \rightarrow K^{-} K^{+}$plays the role of the decay mode $B_{s} \rightarrow \pi \pi$ of case 11 (compare Eqns.(15) and (27)). Thus, as in case 1, we can determine $\gamma$, including all rescattering effects, by measuring the 8 decay modes: $B^{+} \rightarrow \pi^{+} \pi^{0}, B_{d}$ and $\bar{B}_{d} \rightarrow \pi K$ (all), $B_{d} \rightarrow K^{+} K^{-}, B_{d} \rightarrow \pi^{0} \pi^{0}$ and 
$B_{d} \rightarrow \pi^{-} \pi^{+}$(or CP-conjugates of the last three modes). If the annihilation amplitudes are small, as in case 1, we can determine $\gamma$ by measuring any one $B_{d} \rightarrow \pi \pi$ decay mode, in addition to the $B^{+} \rightarrow \pi^{+} \pi^{0}, B_{d}$ (and $\left.\bar{B}_{d}\right) \rightarrow \pi K$ decay modes. As in case 1, if we measure the CP-conjugate $B_{d} \rightarrow \pi \pi$ rates as well, then a CP-averaged measurement of the decay rate $B_{d} \rightarrow K^{+} K^{-}$ suffices.

\subsection{Case 4}

The expressions for the decay amplitudes for $B_{s} \rightarrow \pi^{+} K^{-}$and $B_{s} \rightarrow \pi^{0} \bar{K}^{0}$ in terms of the $S U(3)$ invariant amplitudes are identical to those for $B_{d} \rightarrow$ $\pi^{-} K^{+}$and $B_{d} \rightarrow \pi^{0} K^{0}$, respectively, including annihilation contributions (unlike the case of $B_{d} \rightarrow \pi \pi$ and $B_{d} \rightarrow \pi K$ decays), modulo the CKM factors [8]. Thus, the same method predicts the rates for the $B_{s} \rightarrow \pi K$ decays and the CP-conjugate processes. It suffices to use the $B_{s} \rightarrow K^{-} \pi^{+}$decay (or its $\mathrm{CP}$-conjugate) which is a "self tagging" mode or the $\mathrm{CP}$-averaged decay rate for $B_{s} \rightarrow \pi^{0} \bar{K}^{0}$. Thus, no external tagging is required.

\section{Discussions}

The analysis of the above three cases is strictly valid only in the flavor $S U(3)$ limit. In the tree level amplitudes, i.e., $C_{15}^{T}$ and the $\tilde{T}$ 's, the corrections due to $S U(3)$ breaking can be taken into account more reliably in the factorization approximation and are expected to be given by $f_{K} / f_{\pi}$ (see, for example, Gronau et al. in [1] [10]). For example, in Fig.2 the lengths of $A F, F E$ and $F C$ will have to be multiplied by $f_{K} / f_{\pi}$. However, since the strong penguin amplitudes include $(V-A)(V+A)$ type operators, the (factorizable) corrections due to $S U(3)$ breaking there are less certain, but the corrections are still less than $\sim O(30 \%)$. This is especially relevant for the cases 1 and 2 where the penguin contribution dominates in the $B_{d} \rightarrow \pi K, B_{s} \rightarrow K^{+} K^{-}$ decays and we are predicting this contribution from the $B_{d} \rightarrow \pi \pi$ decays 
using $S U(3)$ symmetry. In the cases 3 and 4 , we use $S U(3)$ symmetry to predict the penguin contribution in a $B_{d} \rightarrow \pi \pi$ (or a $B_{s} \rightarrow \pi K$ ) decay from the $B_{d} \rightarrow \pi K$ decays, but now the tree level contributions dominate the $B_{d} \rightarrow \pi \pi$ decay rate and so the uncertainty due to the $S U(3)$ breaking in the penguin amplitudes is less important.

We have a prediction for more than one rate in some of the cases. For example, in case 1 , we can predict both $B_{s} \rightarrow \pi^{+} K^{-}$and its CP-conjugate decay rate or in case 1, neglecting annihilation, we can predict the decay rates $B_{d} \rightarrow \pi^{-} K^{+}$and $\pi^{0} K^{0}$. So, we can treat the $S U(3)$ breaking in the penguin amplitudes as an unknown and determine it (in addition to $\gamma$ ) from the measurement of seven decay rates.

We have also assumed that the $S U(3)$ breaking in the strong phases is small. A possible justification is that at the energies of the final state particles $\sim m_{b} / 2$, the phase shifts are not expected to be sensitive to the $S U(3)$ breaking given by, say, $m_{K}-m_{\pi}$ (which is much smaller than the final state momenta). However, it is hard to quantify this effect.

If we measure all the $B \rightarrow \pi \pi$ and $B_{d} \rightarrow \pi K$ decay rates, then we can compute the tree level parts of the amplitudes, both $\tilde{T}$ and $\tilde{T}^{\prime}$ (see Eqns.(9) and (22)), as functions of $\gamma$ as discussed in sections 2 and 3 . If the annihilation amplitudes are small, then we have $\tilde{T}=\tilde{T}^{\prime}$ (in the $S U(3)$ limit) since the decay amplitudes $B_{d} \rightarrow \pi \pi$ and $B_{d} \rightarrow \pi K$ are the same up to CKM factors. To include the $S U(3)$ breaking in this analysis, we use the modified relations $C_{15}^{T}(\Delta S=1)=f_{K} / f_{\pi} C_{15}^{T}(\Delta S=0)$ and $\tilde{T}^{\prime}=f_{K} / f_{\pi} \tilde{T}$. This can be used to determine $\gamma$, including $S U(3)$ breaking (without having to to deal with $S U(3)$ breaking in the penguin amplitudes and in the strong phases).

\section{References}

[1] M. Gronau, O.F. Hernández, D. London, J.L. Rosner, Phys. Rev. D52, 6374 (1995). 
[2] N.G. Deshpande, X-G. He, Phys. Rev. Lett. 75, 3064 (1995).

[3] M. Gronau, J.L. Rosner, Phys. Rev. D53, 2516 (1996).

[4] M. Neubert, J.L. Rosner, Phys. Rev. Lett. 81, 5076 (1998).

[5] See, for example, M. Neubert, Phys. Lett. B424, 152 (1998); M. Gronau, J.L. Rosner, Phys. Rev. D58, 113005 (1998).

[6] A.J. Buras, R. Fleischer, hep-ph/9810260.

[7] M. Gronau, D. Pirjol, hep-ph/9811335.

[8] See, for example, A.S. Dighe, Phys. Rev. D54, 2067 (1996).

[9] M. Neubert, J.L. Rosner, Phys. Lett. B441, 403 (1998).

[10] See, for example, H.J. Lipkin, Y. Nir, H.R. Quinn, A.E. Snyder, Phys. Rev. D44, 1454 (1991); M. Gronau, O.F. Hernández, D. London, J.L. Rosner, Phys. Rev. D50, 4529 (1994).

[11] M. Gronau, D. London, Phys. Rev. Lett. 65, 3381 (1990). 